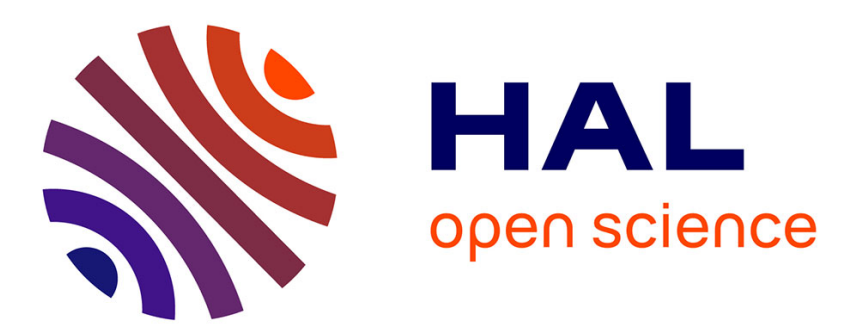

\title{
Habiter un camp de travailleurs. Appropriation, usages et valeurs du dortoir en milieu contraint
}

\author{
Tristan Bruslé
}

\section{To cite this version:}

Tristan Bruslé. Habiter un camp de travailleurs. Appropriation, usages et valeurs du dortoir en milieu contraint. Annales de géographie, 2015, 702-703, pp.248-274. 10.3917/ag.702.0248 . halshs01694824

\section{HAL Id: halshs-01694824 \\ https://shs.hal.science/halshs-01694824}

Submitted on 13 Feb 2018

HAL is a multi-disciplinary open access archive for the deposit and dissemination of scientific research documents, whether they are published or not. The documents may come from teaching and research institutions in France or abroad, or from public or private research centers.
L'archive ouverte pluridisciplinaire HAL, est destinée au dépôt et à la diffusion de documents scientifiques de niveau recherche, publiés ou non, émanant des établissements d'enseignement et de recherche français ou étrangers, des laboratoires publics ou privés. 


\title{
Habiter un camp de travailleurs. Appropriation, usages et valeurs du dortoir en milieu contraint
}

\author{
Living in a labour camp. Appropriation, use and values \\ of the dormitory in a constrained space
}

\section{Tristan Bruslé}

Centre d'études himalayennes, CNRS

Résumé Le fonctionnement des économies des pays du Golfe repose sur le travail des étrangers, qui forment $85 \%$ de la population au Qatar. Parmi ceux-ci, une grande partie des migrants peu payés sont logés dans des camps de travailleurs (labour camp), une forme de "logement contraint ( Bernardot, 2007) correspondant à une gestion spatiale de la main-d'œuvre basée sur des stéréotypes ethniques et sociaux. Les migrants, principalement sud-asiatiques, sont ainsi logés à la périphérie des villes, dans les zones industrielles où sont situés les camps de travailleurs, un dispositif au cœur de la politique migratoire. Dans un contexte contraint, où l'enfermement signifie surtout une impossibilité de se mouvoir hors des zones industrielles, les hommes parviennent néanmoins à habiter leur dortoir. Ils " font avec » les contraintes institutionnelles en employant les tactiques et bricolages que les faibles ont à leur disposition (De Certeau, 1990). Les dortoirs, lieux de vie principaux hors du travail, sont l'objet d'appropriations fines mais indispensables, qui forment autant de micro-résistances à la dépersonnalisation des migrants. Mais le repli sur le dortoir correspond aussi au souhait des autorités de nier toute ambition politique aux migrants considérés comme de passage.

Abstract

Persian Gulf economies rely on foreign workers, who make up $85 \%$ of the total population in Qatar. Among the latter, lowly migrants live in labour camps, a form of "constrained lodging" (Bernardot, 2007) corresponding to a spatial management of migrants based on strong social and ethnic stereotypes. Migrants, most of them South Asians, thus live in the towns' suburbs designated as industrial areas where the camps are situated. These camps are a device at the heart of Qatari migration policy. In a constrained context, where confinement above all means an impossibility to move out of the industrial areas, men manage to make home in the dormitory where ten to twelve of them live together. They "make do with" institutional constraints by using tactics and do-it-yourself solutions the weak have at their disposal (de Certeau, 1990). Migrants appropriate dormitories, the main living spaces away from work, in subtle though indispensable ways. These appropriations are like micro resistances to lack of concern about migrants'wellbeing and living conditions on the part of the authorities. However, the retreat into the dormitory also conveniently meets the wishes of the Qatar State to quash all political aspirations of migrants considered only as a temporary imported workforce.

Mots-clés espace intérieur, appropriation, enfermement, relégation, bricolage, migration, camp 
inner space, appropriation, confinement, relegation, do-it-yourself solutions, migration, work camp

Héritiers des pratiques ségrégationnistes étatsuniennes et importés par l'entreprise pétrolière Aramco en Arabie Saoudite à la fin des années 1930 (Vitalis, 2009), les camps de travailleurs (labour camp) du Golfe persique sont le lieu de résidence de millions de migrants peu qualifiés ${ }^{1}$. Les fonctions du camp répondent à trois objectifs, en fonction des acteurs impliqués. Pour l'État qatarien, redevable à sa population malgré son caractère autoritaire (Heeg, 2010), le camp participe du dispositif de surveillance, de contrôle et de cantonnement des migrants peu qualifiés $^{2}$. Pour les entreprises qui emploient les ouvriers, il permet une mise à disposition de la main-d'œuvre nombreuse qu'il s'agit alors uniquement de transporter sur son lieu de travail. Enfin, pour les principaux concernés, le camp est l'espace imposé de la vie quotidienne. À ce titre, il est un système de mise à l'écart ou d'enfermement d'individus considérés comme indésirables, mais néanmoins désirés pour leur force de travail.

Espaces à l'écart de la société qatarienne, les camps de travailleurs et les zones industrielles où ils sont situés participent donc d'un système de gestion des autres, les migrants en l'occurrence, placé sous le signe du confinement spatial. Celui-ci est une technique utilisée pour gérer des catégories et des territoires considérés comme problématiques (Goffman, 1968 ; Foucault, 1975 ; Wacquant, 2010 ; Milhaud, 2009). Il s'insère aussi dans un système de migrations internationales temporaires où les migrants sont plus considérés comme une force de travail que comme des individus ${ }^{3}$. L'immigration sans intégration caractéristique des pays du Golfe (Beaugrand, 2010) a comme corollaire un logement des ouvriers ne favorisant pas l'enracinement et poussant au contraire à la rotation des migrants. La question d'un habiter à la marge de l'habiter classique, en termes d'appropriation ou de relation à l'espace par exemple, se pose alors. À l'échelle micro de l'intérieur de ces camps, les adaptations des migrants sont autant de résistances à l'ordre imposé (Goffman, 1968), qui se matérialisent par une appropriation fine de l'espace. Les questions d'intimité, d'espace personnel, de limites entre les retenus sont posées car le confinement exacerbe le rapport qu'ont les hommes à la distance (Sibley et Van Hoven, 2009 ; Baer, 2005 ; Moran, Pallot et Piacentini, 2013 ; Martin et Mitchelson, 2009). En situation de forte densité et sous contrainte, la production de l'espace du quotidien

1 L'auteur remercie Marie Morelle, Djémila Zeneidi et les deux relecteurs anonymes pour leurs remarques avisées, ainsi que Laetitia Perrier Bruslé pour la mise en forme des figures. Je traduirai labour camp par camp de travailleurs. Les termes de foyers pour travailleurs, résidences ou logements traduisent mal le caractère éminemment provisoire du camp tel qu'il est pensé par ses promoteurs. Enfin, le labour camp est un camp de travailleurs ou pour travailleurs et non un camp de travail, car il ne sert qu'au repos des migrants. Le travail est effectué sur les chantiers de construction, dans les bureaux ou les commerces.

2 De la même manière les centres de rétention et parfois les prisons sont des instruments de gestion de la migration internationale.

3 Cela correspond à ce que dit Castles $(2006,742)$ à propos des politiques migratoires européennes de 1945 à 1970 : « import labour but not people ». 
exprime des rapports de pouvoir entre des structures dominantes et des hommes poursuivant des stratégies d'adaptation, souvent à grande échelle. Étonnamment, la géographie ne s'intéresse que rarement aux micro-espaces (Staszak, 2001), encore moins aux espaces intérieurs, alors que l'étude de l'habitat rural, comme expression culturelle, a longtemps été l'apanage des géographes ruraux. L'ouvrage « Espaces domestiques. Construire, habiter, représenter » (Collignon et Staszak, 2003) a posé une pierre dans l'édifice de la micro-géographie mais il semble qu'en dehors de problématiques domestiques, les géographes ont peu suivi cette initiative. Les questions d'intimité et d'espace intérieur dans la rue ont néanmoins été étudiées par Morelle (2006). La géographie des intérieurs reste donc un domaine en friche qui devrait être étudié de l'intérieur certes (Staszak, 2001) mais aussi en s'intéressant aux forces qui les façonnent de l'extérieur. Celles-ci sont particulièrement actives dans le contexte du Qatar où le "logement contraint », qui « recouvre des espaces désignés institutionnellement pour être affectés de manière provisoire à des fonctions d'accueil et de résidence de populations définies comme indésirables et inutiles » (Bernardot, 2007) est la forme la plus commune d'habitation pour les migrants peu qualifiés. Je m'intéresse donc ici à l'exploration de la production de l'espace «par le bas », c'est-à-dire par des migrants acteurs eux aussi d'une forme de mondialisation (Portes, 1997). Dans cette perspective de l'étude d'un lieu non porteur de valeurs transcendantes, le dortoir est une unité facilement préhensible. Elle nécessite une analyse à l'échelle du corps, puisque l'intérieur se mesure en mètres, parfois en centimètres et un protocole de recherche qui permette l'entrée physique du chercheur dans son objet. L'espace que je vais tenter d'appréhender ici n'est pas tout à fait du ressort d'une géographie domestique, ni du domaine strictement privé mais concerne bien une géographie de l'intérieur.

Dans le contexte d'une privation d'espace et de mouvement, même si elle n'équivaut jamais à celle de la prison, la question de la production de l'espace par les individus se pose à la fois en termes matériels, relationnels et idéels. L'étude des espaces intérieurs des camps pose ainsi la question de l'habiter, comme pratique et mise en signification des lieux (Stock 2004), dans le cadre d'une biopolitique où le logement répond à des objectifs de production ${ }^{4}$. Entre camp de rétention et logement pour ouvriers, comment le camp de travailleurs est-il habité ? Comment produire et s'approprier un espace contraint ${ }^{5}$ ? En quoi l'espace du dortoir reflètet-il des conditions d'enfermement ? Quelle est sa valeur ? Le dortoir, espace à la fois clos et ouvert mais bien délimité, permet des observations des aménagements sur la durée, sur les «manières de faire » (de Certeau, 1990) des hommes dans

4 La biopolitique est ici envisagée comme l'ensemble des moyens d'action sur une catégorie de population, destinés à maximiser les forces productives de celles-ci. Elle passe par une politique spatiale et hygiéniste dont les corps des migrants sont les objets.

5 L'appropriation de l'espace a une double dimension idéelle et matérielle (Di Méo, 1998) et rappelée par Ripoll et Veschambre $(2005,10)$ pour qui « penser en termes d'appropriation de l'espace conduit à envisager l'occupation ou l'usage de l'espace, mais aussi sa production et son détournement, son marquage, sa valorisation ou inversement sa stigmatisation ». 
la banalité de leur vie quotidienne. Le dortoir peut alors être étudié comme « un espace de jeu pour des manières d'utiliser l'ordre contraignant du lieu » (de Certeau, 1990, 77). Cet espace de jeu est avant tout un espace de négociation, d'arrangement et pour tout dire de « bricolage » (Lévi-Strauss, 1962), c'est-à-dire d'inventions manuelles et de moyens détournés qui ne sont pas sans rapport avec la ruse. La contrainte spatiale pousse en effet les travailleurs à faire eux-mêmes (non à faire faire) avec les «moyens du bord » (ibid., 27). Les ruses, les tactiques que le faible a à sa disposition (de Certeau, 1990) donnent lieu à un ensemble de créations minutieuses qui prennent corps dans l'espace. Derrière l'invisibilisation voulue des migrants, une vie sociale est à l'œuvre : mon objectif est aussi de montrer les stratégies des acteurs et de combattre l'aspect caché des centres de confinement (Martin et Mitchelson, 2009) pour les faire apparaître comme des lieux où les enfermés mettent en ouvre des tactiques.

Cet article est basé sur des enquêtes et des observations effectuées au Qatar dans le cadre du programme TerrFerme ${ }^{6}$. Au total, j'ai passé presque trois semaines dans le camp Al Mihinat ${ }^{7}$, quelques jours dans un autre, en plus de visites ponctuelles dans les camps des différentes zones industrielles du Qatar. La majorité d'entre eux était occupée par des travailleurs migrants népalais dont je parle la langue. Dans tous les cas, les observations que j'ai menées l'ont été en accord avec les hommes.

Après avoir vu comment le camp est un dispositif majeur de la politique migratoire qatarienne, je montrerai comment la situation d'enfermement conditionne la manière dont les hommes s'approprient l'espace du dortoir.

\section{Le camp de travailleurs au cœur de la politique migratoire}

Au Qatar comme dans les autres micro-États du golfe arabique, l'économie repose sur le travail des étrangers. Ceux-ci représentent $94 \%$ des employés, dont quasiment $100 \%$ dans le secteur privé (Baldwin-Edwards, 2011). Parmi les 1,9 million d'habitants en 2013, environ 220000 sont Qatariens et disposent de tous les avantages d'un État providence très généreux avec ses citoyens. A contrario, la politique migratoire du Qatar est placée sous le signe de la non-assimilation des migrants, l'acquisition de la nationalité qatarie étant impossible ${ }^{8}$. Les migrants ont des statuts sociaux variés, les travailleurs peu qualifiés, principalement originaires d'Asie du Sud, formant la majorité d'entre eux ${ }^{9}$. La «violence structurelle»

6 Le programme de recherche TerrFerme «Les dispositifs de I'enfermement. Approche territoriale du contrôle politique et social contemporain », est financé par l'ANR (Réf. : ANR-08-JCJC 2008-0121-01) et le Conseil Régional d'Aquitaine (Réf. : 2010407003).

7 Le nom du camp et celui des migrants ont été changés. Ma première visite dans le camp date de 2006. J'y suis retourné en 2008 et deux fois en 2011.

8 Il existe des exceptions pour les sportifs de haut niveau.

9 Les statistiques qataries sont difficiles à traiter, compte tenu des catégories employées et de la nonexhaustivité des chiffres. On peut estimer qu'environ 80 \% des travailleurs étrangers sont peu qualifiés 
(Gardner, 2010a) de la gestion des travailleurs étrangers se fait au sein du système du parrainage (sponsorship). Le parrain est un citoyen qatarien qui donne à un immigré le droit de travailler et de résider au Qatar. Ce droit se paie et se traduit par une soumission et une dépendance totales du migrant vis-à-vis du parrain, notamment pour le travail qu'il est impossible de quitter sans son accord. Il est aussi habituel que le parrain confisque le passeport du migrant qui de toute façon ne peut sortir du pays sans l'accord écrit du sponsor. Ce système de contrôle des migrants prend une dimension spatiale grâce au logement.

\subsection{La ségrégation spatiale des migrants peu qualifiés et le modèle du camp}

Si la ségrégation n'est pas un principe nouveau à Doha (Wiedmann, Salama, et Thierstein, 2012), l'explosion du nombre de migrants, peu payés en particulier, a entraîné un zonage et une division de l'espace de plus en plus fondés sur des bases nationales et sociales. Cela s'est aussi fait de manière aléatoire ou peu contrôlée (Adham, 2008). La répartition des individus dans l'espace répond ici aussi à la règle des «emplacements fonctionnels» (Foucault, 1975, 168) qui crée un espace utile, celui du logement des forces de production. Les quatre grandes zones industrielles (industrial areas) et les multiples camps disséminés dans le pays hébergent en 2013 au moins un million d'hommes et dans une moindre mesure, de femmes, dans un environnement opposé au Doha rutilant et clinquant ${ }^{10}$. Elles regroupent un ensemble d'activités considérées comme polluantes: des usines de transformation, des garages, des ateliers et des logements pour travailleurs (labour camps). Bien qu'elles soient considérées comme dégradantes par leurs habitants, elles sont le lieu de vie principal des migrants à bas revenus. Leur aspect désolé et peu entretenu témoigne d'un abandon et d'un désintérêt pour la vie des hommes qui vivent au milieu du bruit des norias de camions et de la poussière qu'ils soulèvent. Ces zones industrielles répondent à une utilisation de l'espace comme moyen de contrôle de populations considérées comme menaçantes pour l'identité nationale, les femmes et la société dans son ensemble (Heeg, 2010). Elles délimitent une géographie de l'enfermement et traduisent la volonté de confinement et de relégation des ouvriers pauvres et étrangers. Ce confinement est à la fois une pratique spatiale et le lieu d'une production discursive qui s'alimentent l'une l'autre ${ }^{11}$. Comme

et que $50 \%$ d'entre eux travaillent comme ouvriers dans le bâtiment (calculs d'après les chiffres de Qatar Statistical Authority).

10 Trois grandes zones industrielles sont fermées aux visiteurs car ce sont des sites proprement industriels : Ras Laffan, Messaieed et Dukhan (respectivement à 85, 40 et 80 kilomètres de Doha). Celle de Doha, dans laquelle habite une grande partie des migrants non qualifiés au Qatar, est située à une quinzaine de kilomètres du centre-ville. Si l'entrée dans cette dernière zone est libre, certains camps sont surveillés par des gardiens.

11 Une femme qatarienne interrogée à propos de la présence de célibataires (bachelor est le terme en vigueur pour désigner un travailleur migrant) dans son quartier assure que « la présence de célibataires dans des quartiers familiaux est source de risques. Comme ils sont de nationalité et de culture différente, ils pourraient ne pas s'adapter à la nature conservatrice de la société qatarienne » (Qatar Tribune, 13 décembre 2012). 
l'a remarqué Sayad (1980), les représentations de l'immigré influent sur le statut du logement qui lui-même participe des perceptions culturalistes à l'égard de l'immigré. À l'échelle d'étude des banlieues européennes et des centres-villes étatsuniens, Wacquant (2007) montre comment stigmatisation symbolique et territoriale se nourrissent pour aboutir à une marginalité avancée. Au Qatar, à cause de l'existence de processus discursifs de même type, l'ouvrier célibataire sud-asiatique est physiquement éloigné du reste de la société et sa vie est presque cantonnée aux camps ${ }^{12}$.

Le camp de travailleurs est une forme de «logement contraint » (Bernardot, 2007) loué par l'employeur pour héberger ses travailleurs, qui n'ont d'autres choix que d'y vivre. Comme tel fait partie d'un dispositif spatial de mise à l'écart des migrants. Il est associé à des programmes d'accueil temporaire de travailleurs migrants, dont la gestion repose sur le court séjour. Ailleurs dans le monde, le logement en camp a été utilisé en Afrique du Sud pour séparer les populations et reléguer les Noirs au sein d'un système de discrimination et d'exploitation économique (Ramphele, 1993). Aujourd'hui c'est sans doute une forme de logement en expansion, dont les degrés d'enfermement et de contrainte sont variables. Au Dakota du Nord (États-Unis) ou en Alberta (Canada), les travailleurs se regroupent volontairement dans des man camps, à proximité des sites de production de pétrole et de gaz de schiste (Caraher, à paraittre). En Espagne, le camp est utilisé pour loger d'autorité les migrantes au statut temporaire venues pour la récolte des fraises (Zeneidi, 2011). En Chine du sud, le système du dortoir pour travailleurs (dormitory labour regime) s'insère dans la nouvelle division internationale du travail qui conduit à des formes de contrôle de la main-d'œuvre migrante dont le dortoir est une base essentielle la pierre angulaire (Pun et Smith, 2007). En même temps, le dortoir devient le lieu où les revendications des ouvriers, comme dans le cas des usines Foxconn fournisseuses d'Apple (Pun et Chan, 2013), donne lieu à la création d'une nouvelle classe ouvrière (Chan et Ngai, 2009).

\subsection{Le camp, un espace de vie fonctionnel}

Le logement en labour camps est un fait majeur au Qatar. En 2010, selon les statistiques qatariennes, 920000 personnes habitaient de tels logements, soit $62 \%$ de l'ensemble des étrangers ${ }^{13}$. Le mot camp est entré dans le vocabulaire des migrants et forme ainsi une réalité incontournable du cantonnement, vécue au quotidien par les migrants. En tant que « logement provisoire pour des travailleurs provisoires » (Sayad, 1980), le camp de travailleurs est délaissé par les autorités qatariennes mais il est central pour éloigner les migrants. Les réglementations en

12 Le vendredi, unique jour de congé de la semaine, les principaux lieux de divertissement de Doha (les centres commerciaux, le zoo, la promenade du bord de mer à Doha, les parcs publics, le faux vieux souk) sont interdits aux célibataires, pour cause de "jour de la famille ». Cette règle vise de fait les célibataires sud-asiatiques.

13 Le nombre de camp de travailleurs est inconnu. 
vigueur concernant le nombre de personnes par chambre ou le nombre de mètres carrés par travailleur sont quasi systématiquement violées. Le faible nombre d'inspecteurs du travail (150 pour 1,2 million de travailleurs migrants en 2013), qui s'occupent aussi des conditions de logement mais ne parlent pas les langues des travailleurs, rend leur action dérisoire (Human Rights Watch, 2012). Alors qu'il est très répandu dans le Golfe, le labour camp en tant que tel n'a fait l'objet que de rares publications, à l'exception de Gardner (2010b) qui en distingue quatre types en fonction de leur degré d'organisation ${ }^{14}$. Dans les camps de grande taille (plusieurs milliers de migrants), le logement est divisé par nationalité et en fonction de la position hiérarchique des hommes dans l'entreprise. Le degré d'entassement dans les mobiles homes, le droit à des cantines de qualité variable suivent aussi ces règles hiérarchiques. Dans tous les cas, les habitants ne sont ni locataires, ni propriétaires et disposent de peu de droits. Leur séjour est vu comme provisoire même si certains habitent le même camp depuis dix ans. C'est donc un modèle de logement que tout éloigne du paternalisme, tel qu'il a pu être pratiqué en Europe. Il n'existe aucune volonté d'éducation, de formation ou d'apprentissage à l'hygiène. C'est une forme basique de gestion de la main-d'œuvre où la récupération des forces est au centre des préoccupations des employeurs. Il est l'unité spatiale de base du dispositif de cantonnement des travailleurs migrants.

\subsubsection{Géographie du camp Al Mihinat}

Le camp Al Mihinat accueille deux cents Népalais, originaires de plusieurs régions du Népal, et une petite dizaine d'Indiens ${ }^{15}$. En majorité non mariés, $60 \%$ des hommes ont entre 20 et 29 ans. Ils sont employés par un prestataire de services qui les fait travailler dans les bureaux d'une grande entreprise qatarienne de gaz et les logent. Le seul espace commun à tous les hommes du camp, en dehors des espaces extérieurs de parking des bus où des parties de volley-ball sont organisées, est la petite cantine où sont servis les repas et où est diffusée une chaîne de télévision népalaise. Deux autres mobiles homes sont investis par une association culturelle créée par des migrants, mais tous n'en font pas partie ${ }^{16}$. Pensé pour le logement, le camp offre donc peu d'espaces de sociabilité hors des dortoirs (Figure 1).

Si les espaces intérieurs et les dortoirs sont bien entretenus, les douches et le couloir principal sont assez sales, malgré le travail de deux hommes chargés de l'entretien du camp. Personne n'a le souvenir d'une quelconque visite

14 Voir aussi Bruslé (2012a) pour une étude de la vie quotidienne dans un camp. Les organisations non gouvernementales comme Human Rights Watch ou Amnesty International I'envisagent d'un point de vue humanitaire ou sous l'angle des droits de l'homme.

15 Voir Bruslé (2010) pour une analyse socio-économique du camp. Les travailleurs indiens et népalais ont des emplois similaires, se nourrissent à la même cantine. Les Indiens occupent néanmoins un dortoir à part.

16 D'autres camps sont uniquement faits de mobiles homes. Les espaces collectifs sont réduits à la portion congrue dans la majorité des camps. 


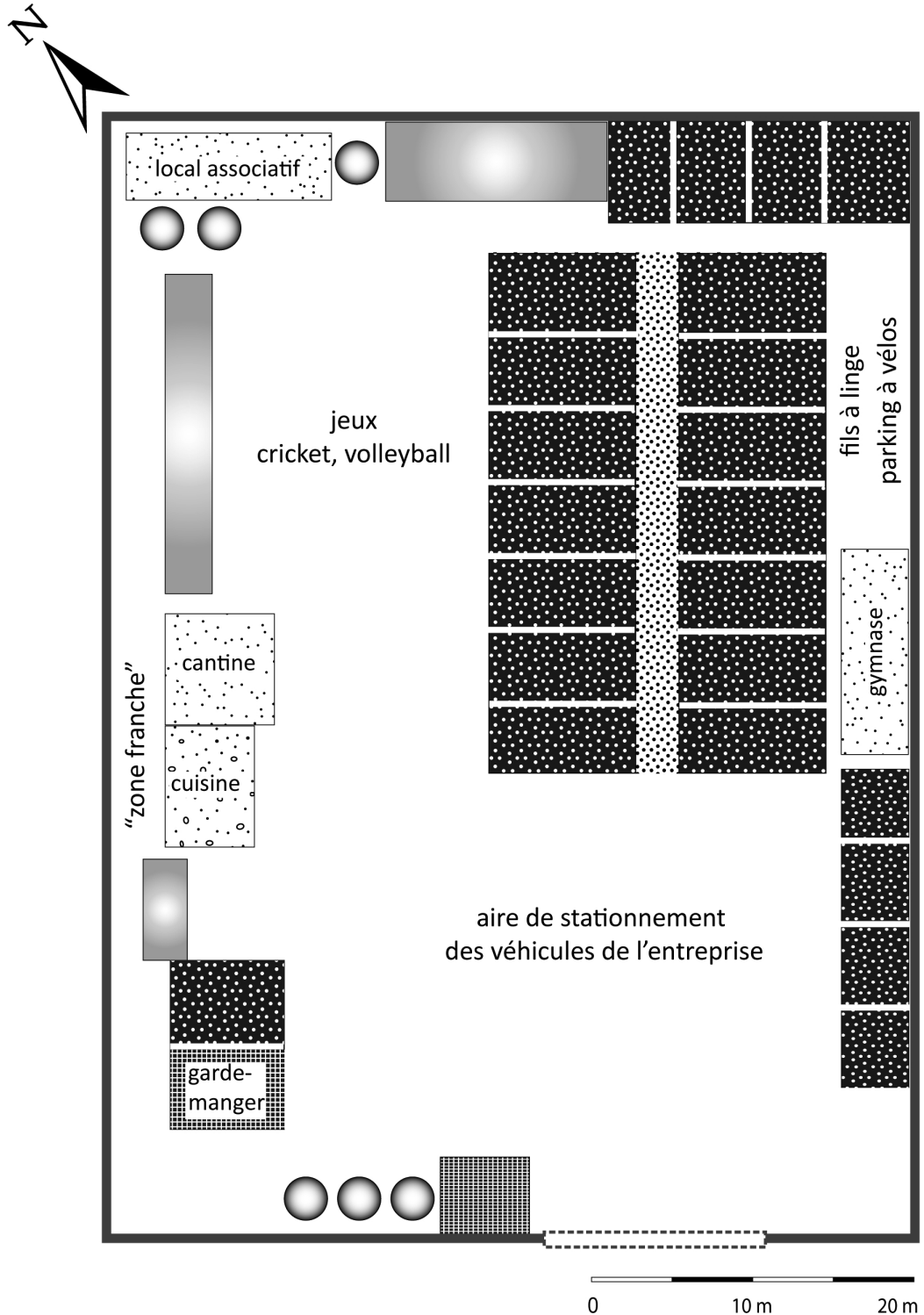

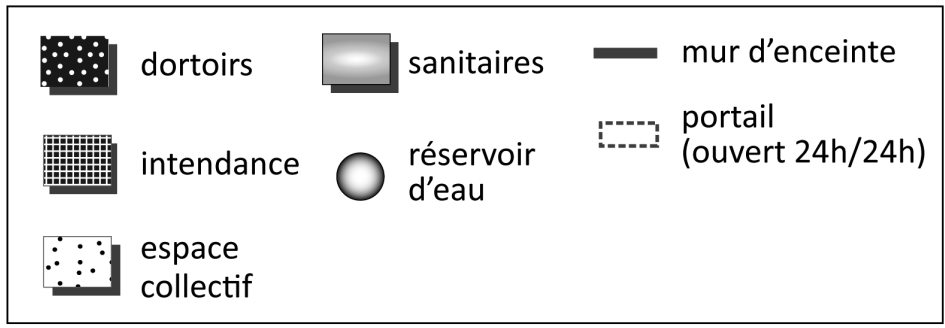

Fig. 1 Les espaces du camp de travailleurs Spaces existing in the labour camps 
d'inspecteurs qatariens alors que le camp pourrait faire l'objet de redressements. Espace ultra-fonctionnel, il ne répond à aucune préoccupation architecturale liée au bien-être de ses habitants. Sous un grand hangar ont été construits, de part et d'autre d'un couloir large de trois mètres, quatorze dortoirs identiques de vingt-huit mètres carrés chacun. Les portes des chambres sont fendues, la peinture s'écaille et partout la poussière vole. Au fur et à mesure des recrutements, huit autres chambres ont été ajoutées, dont quatre dans deux mobiles homes. Parce qu'il est pensé comme provisoire, le camp ne bénéficie que de peu d'amélioration au fil des ans. Entre 2008 et 2011 , seule la cantine a été refaite et agrandie. Les investissements des logeurs dans le camp sont donc minimaux, en particulier dans les dortoirs.

L'architecture ne peut donc pas être considérée comme disciplinaire. D'une manière générale la surveillance, normalement assurée par un contremaître (qui loge ailleurs) et deux camp boss (qui logent sur place), est quasi inexistante.

\subsubsection{Les circulations dans le camp}

Dans le camp Al Mihinat, les hommes peuvent circuler librement partout, sauf dans le garde-manger dont les cuisiniers gardent la clép ${ }^{17}$. Hormis cette exception, les « d'effets de seuil » tels ceux qui existent en prison ou en centre de rétention (Goffman, 1968 ; Morelle, 2013 ; Milhaud, 2009 ; Michalon, 2012) sont peu affirmés. Tous les hommes n'entrent pas dans le local de l'association culturelle qui gère aussi une salle de musculation, produit des concerts et organise des rencontres littéraires. Sa dizaine de membres utilise donc de façon quasi exclusive le mobile home qui lui sert de base. En outre, il y existe dans le camp des espaces cachés, mais connus de tous, où les travailleurs se livrent à des activités interdites (voir Figure 1). Derrière le bâtiment abritant le réfectoire, là où les hommes ont à disposition un espace pour faire eux-mêmes la cuisine, quelques sièges en plastique sont disposés autour d'un barbecue de fortune. C'est là que parfois, certains se réunissent pour déguster des brochettes de foie de poulet et surtout pour boire du whisky ${ }^{18}$. Ces «zones franches» (Goffman, 1968, 286) sont des espaces de liberté, " où l'autorité du personnel se fait moins sentir » (ibid. 284), où les migrants peuvent jouer avec les règles du camp en particulier et avec celles du Qatar en général. C'est l'espace des « adaptations secondaires », celles qui permettent «à l'individu d'utiliser des moyens défendus, ou de parvenir à des fins illicites (ou les deux à la fois) et de tourner les prétentions de l'organisation relatives à ce qu'il devrait faire ou recevoir, et partant à ce qu'il devrait être »

17 Le règlement affiché dans le couloir du bâtiment central est peu connu et peu respecté. L'interdiction de boire de l'alcool ou de faire entrer des étrangers dans le camp n'est jamais suivie. Au niveau national, il n'existe pas de lois spécifiquement destinées aux habitants des camps.

18 Les étrangers gagnant plus de 4000 Riyals $(830 €)$ par mois peuvent, après l'obtention d'une licence, acheter de l'alcool dans I'un des deux magasins contrôlés par l'État au Qatar. Les ouvriers, dont les salaires sont compris entre $180 €$ et $300 €$ sont de fait exclus par cette mesure. Ils se tournent alors vers le marché noir, boivent du parfum en vente libre dans toutes les échoppes des zones industrielles ou achètent de l'alcool fabriqué artisanalement dans les camps. 
(Goffman, 1968, 245). Mais l'ambiguïté de la discipline du camp, comme dans l'asile où «le personnel rend possible l'accès à des zones interdites» (ibid. 285) fait que l'idée d'une transgression de règles est peu présente à l'esprit des buveurs. La consommation d'alcool se fait hors du champ visuel mais au su de tous, encadrants y compris. Au Népal aussi, la consommation d'alcool étant mal considérée, il faut se cacher pour boire : le camp reproduit dans une certaine mesure les conditions de l'alcoolisation habituelles, celles de la société d'origine.

Les liens entre l'intérieur et l'extérieur du camp sont forts car les circulations ne sont pas contrôlées ${ }^{19}$. Les hommes peuvent sortir comme ils le veulent, s'approvisionner en nourriture dans les boutiques de la zone industrielle ou aller surfer sur internet dans les cybercafés, de sorte que le sentiment d'enfermement n'est pas lié au camp lui-même sinon aux zones industrielles d'où il est plus difficile de sortir. L'absence de transports publics efficaces, la cherté des taxis clandestins et les restrictions à la fréquentation de certains lieux n'incitent pas les hommes à sortir de leur camp ou de la zone industrielle dans laquelle il est situé. La position d'infériorité et de fragilité du migrant au Qatar, qui n'a aucun droit politique et peut être renvoyé sans délais dans son pays, est en outre parfaitement intégrée par les hommes. Cette position sociale intériorisée va de pair avec l'acceptation d'un cantonnement qui n'existe pas grâce à des barrières physiques ou par l'usage de la force. En la quasi-absence de surveillance, le camp Al Mihinat est presque uniquement un espace de retenus.

\section{Habiter le dortoir, entre contrainte et promiscuité}

La question de l'habiter dans le dortoir d'un camp de travailleurs se pose avec acuité, tant les rapports au lieu sont marqués par la contrainte.

D'un camp à l'autre, l'uniformité des dortoirs est frappante. Derrières les portes donnant sur de longs couloirs, les mêmes room, monotones, souvent sans fenêtre, à l'air saturé d'odeur de cuisine, d'humidité et de renfermé et aérées seulement par une climatisation bruyante. Après avoir laissé derrière soi le couloir poussiéreux, on entre dans le dortoir sans frapper. L'entassement des hommes et des objets saute aux yeux. L'espace du dortoir est à la fois plein et dénué de tout superflu. Dans le camp Al Mihinat chacun des dix hommes dispose en moyenne de $2,8 \mathrm{~m}^{2}$, loin des quatre mètres carrés réglementaires ${ }^{20}$ : l'espace du dortoir est éminemment collectif. L'imposition aux travailleurs d'un style de vie communautaire est-elle liée à des préjugés culturels sur la grégarité

19 Un gardien est resté quelques mois à l'entrée du camp en 2011 mais ses contrôles étaient très limités.

20 Les 20 dortoirs accueillent entre huit et douze hommes, la moyenne étant de dix. Le recensement exhaustif de toutes les chambres montre qu'il n'y a pas d'homogénéité de castes mais que le regroupement se fait plutôt par date d'arrivée, ce qui signifie aussi souvent qu'ils sont arrivés quasiment ensemble au Qatar, grâce à la même agence de placement ou au même intermédiaire. Au fil des années, au gré des arrivées et des départs, la composition du dortoir change. 
des populations immigrées (Sayad, 1980) ? $?^{21}$ Toujours est-il que les hommes se serrent dans les dortoirs, à l'instar des objets comprimés dans les interstices. Les lits sont superposés, les affaires s'empilent du sol au plafond: les potentialités de la verticalité sont retrouvées pour optimiser la densité par mètre carré. Une économie de la rareté spatiale se met en place.

\subsection{Espace et bricolage : prendre place dans le dortoir}

Contrairement à ce qui se passe en prison, ou dans les centres de rétention, le dortoir du camp de travailleur peut être choisi. Si au départ, le migrant est affecté à un dortoir et à un lit en fonction des disponibilités, il peut ensuite changer de chambre selon son gré, le superviseur ne faisant qu'approuver un choix basé sur des affinités. Il existe donc des « marges de négociation » (Milhaud, 2009 ; Michalon, 2012 ; Darley, 2008) dans l'espace du camp, marges qui concernent les relations sociales et l'espace. À mesure que les hommes prennent place dans leur chambre, qu'ils s'y installent parfois pour des années, le nombre d'objets possédés augmente. Il faut leur trouver un emplacement dans l'espace contraint du dortoir. Les hommes doivent donc retourner la contrainte spatiale, l'optimiser et la rendre vivable en aménageant l'espace. L'appropriation spatiale est "prise de possession » (Veschambre, 2005, 115). Se forger des parcelles d'intimité dans un espace collectif peut être en outre considéré comme une manière de combattre la dépersonnalisation (Goffman, 1968) induite par le travail et la vie dans le camp.

\subsubsection{Les aménagements et le bricolage de l'espace}

Les cinq lits superposés du dortoir sont disposés autour de la pièce de sorte que l'espace central est laissé libre pour la circulation des hommes (Figure 2). Le reste de la pièce est rationalisé, comme dans les dortoirs des prisons où l'on se sert "d'un minimum d'objets et d'une imagination féconde » (Sibley et Van Hoven, $2009,201)$. À part les lits qui sont fournis par l'entreprise, ainsi qu'un mince matelas et une paire de draps, les chambres ne contiennent au départ pas d'autres meubles. Le capital des hommes étant limité et le but ultime de leur séjour étant d'épargner pour " envoyer de l'argent à la maison ", une économie du recyclage et de la récupération se met en place. La moquette ou le linoléum, souvent taché ou moisi, vient des bureaux où travaillent les hommes. Les tables et les chaises ont été données par des employés des entreprises aux travailleurs au moment de leur déménagement. Il en va de même pour les quelques commodes en bois ou armoires métalliques mais en revanche les armoires en plastique sont achetées par les hommes. Tous n'en possèdent pas : c'est un signe de richesse relative et aussi du temps passé au Qatar. Ainsi ce migrant tout juste revenu du Népal sortait avec fierté sa collection de chemises, qu'il suspendait avec soin dans son meuble personnel, où il rangeait aussi son whisky.

21 La difficulté d'enquêter auprès des acteurs institutionnels, qui ne souhaitent pas communiquer sur le sujet des camps de travailleurs, ne me permet pas de rendre compte en profondeur de leur vision des migrants et des camps. 


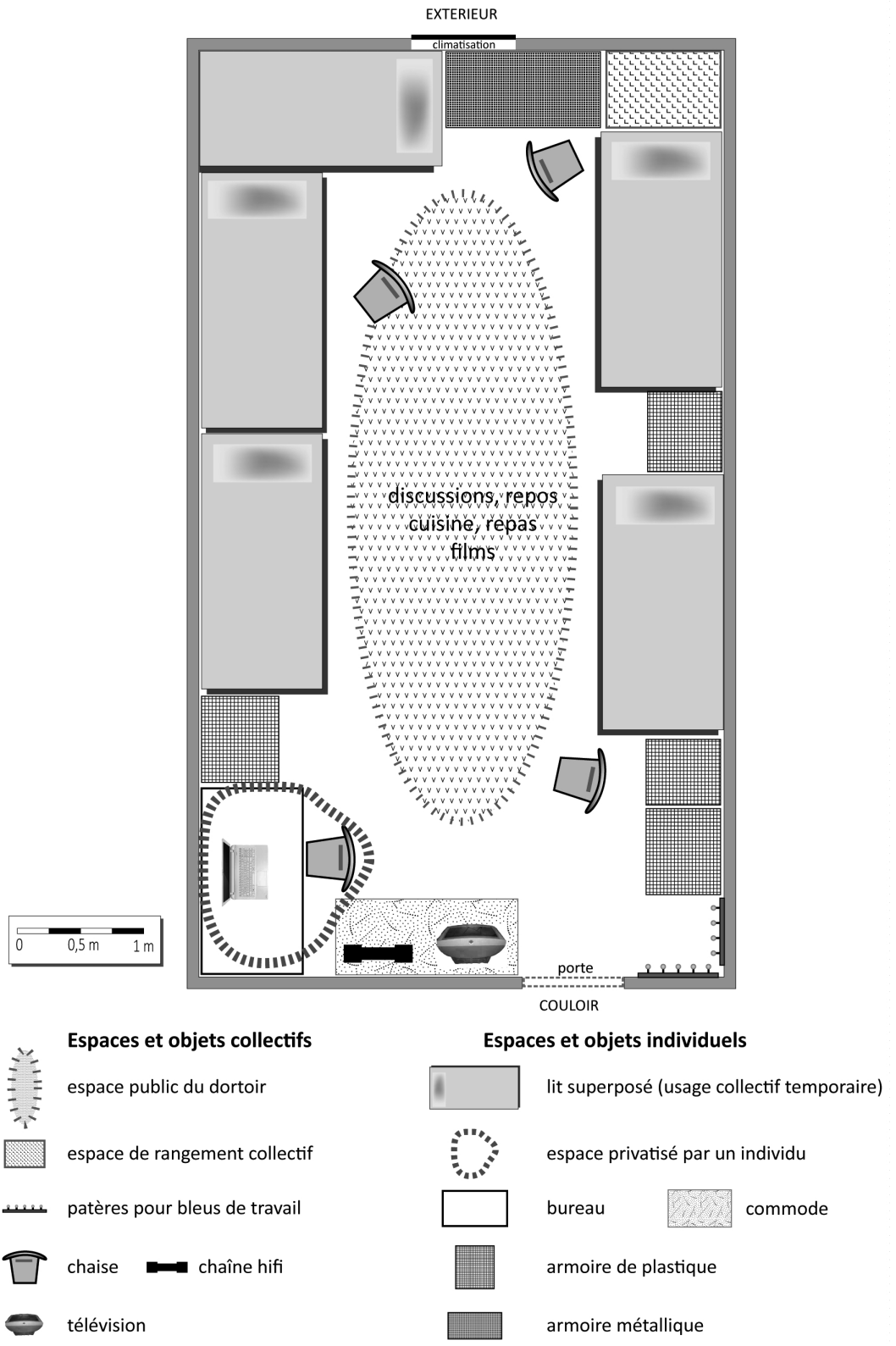

Fig. 2 Les espaces privés et publics du camp de travailleurs Al Mihinat Public and private spaces in Al Mihinat labour camp 
Sur les murs et au plafond courent en tous sens des fils électriques qui se rejoignent aux multiprises et alimentent les nombreux appareils de la chambrée. Chacun tente de se brancher, certains se connectent au réseau internet pour cinq euros par mois, grâce à un fil qui passe par le faux plafond. Il faut se débrouiller, surtout pour trouver de la place. Dans les coins, sous les lits, les valises et les malles sont entassées, souvent au bénéfice de celui qui occupe le lit du bas. Il faut économiser l'espace pour ranger, quitte à bricoler des installations. Certaines sont pérennes, telle l'étagère faite d'une planche qui permet le rangement à hauteur du lit, d'autres sont plus humbles, tels les verres en plastiques scotchés aux pieds du lit pour contenir une brosse à dents et un dentifrice. Sur les murs, entre deux clous, le fil tendu sert à suspendre le linge tandis que la serviette sèche sur les barreaux ou l'échelle du lit. Parfois, pour poser la télévision, un simple carton renforcé par des bouts de bois suffit. Et pour fixer un rideau autour du lit supérieur, les extensions sont faites de baguettes de bois fixés aux montants du lit par du ruban adhésif. Par cet ensemble de petites installations, il s'agit donc de «faire avec » l'espace contraint et dense du dortoir et de produire un espace personnel utile. Il s'agit aussi pour le travailleur devenu bricoleur d'utiliser " un ensemble à chaque instant fini d'outils et de matériaux, hétéroclites » (LéviStrauss, 1962, 27), grâce à la récupération de "résidus d'ouvrages humains » (ibid. 29). Le bricolage correspond en outre à la volonté des hommes de ne pas trop dépenser, de ne pas trop s'investir dans un lieu qui n'est habité que dans la mesure où il sert un autre objectif situé au Népal. Il est une pratique micro-spatiale qui permet de trouver la combinaison la plus apte à répondre à des besoins récurrents, dans une économie d'espace et de geste. Un homme a par exemple judicieusement fixé une planche entre l'étage supérieur du lit où il dort et l'armoire qui lui est adjacente. Il pose ainsi son ordinateur sur cette planche et peut, assis en tailleur et grâce à un clavier mobile, surfer sur internet (Photo 1).

La solution la plus courte face à la rareté spatiale reste l'empilement: la chambre est un espace horizontal peuplé d'objets à la verticalité affirmée. À droite en entrant dans la chambre, derrière la porte, pendent aux patères les bleus de travail des hommes qui laissent ainsi en entrant leur identité d'ouvrier. Il s'agit d'occuper l'espace de haut en bas, dans l'espace imparti par les lits qui forment les limites. Ensuite, on peut «bourrer» entre les lits, occuper les interstices car ici «c'est entassé, pas comme au village ». Certains, les plus anciens occupants ou les opportunistes, s'approprient les coins de la chambre pour y mettre une petite table et privatisent ainsi un espace a priori commun. Un bouquet de fleurs en plastique et un lampion chinois pendu au plafond complètent le décor ainsi personnalisé.

\subsubsection{La décoration et les objets}

Entre mon passage en 2008 et en 2011, de nombreuses chambres ont été repeintes aux frais des occupants, lassés des écailles de peinture et de la moisissure qui se développait sur les murs. Tendre une bâche de plastique sur le mur permet de réduire l'humidité du lit mais n'est pas très pérenne tandis que la peinture, 


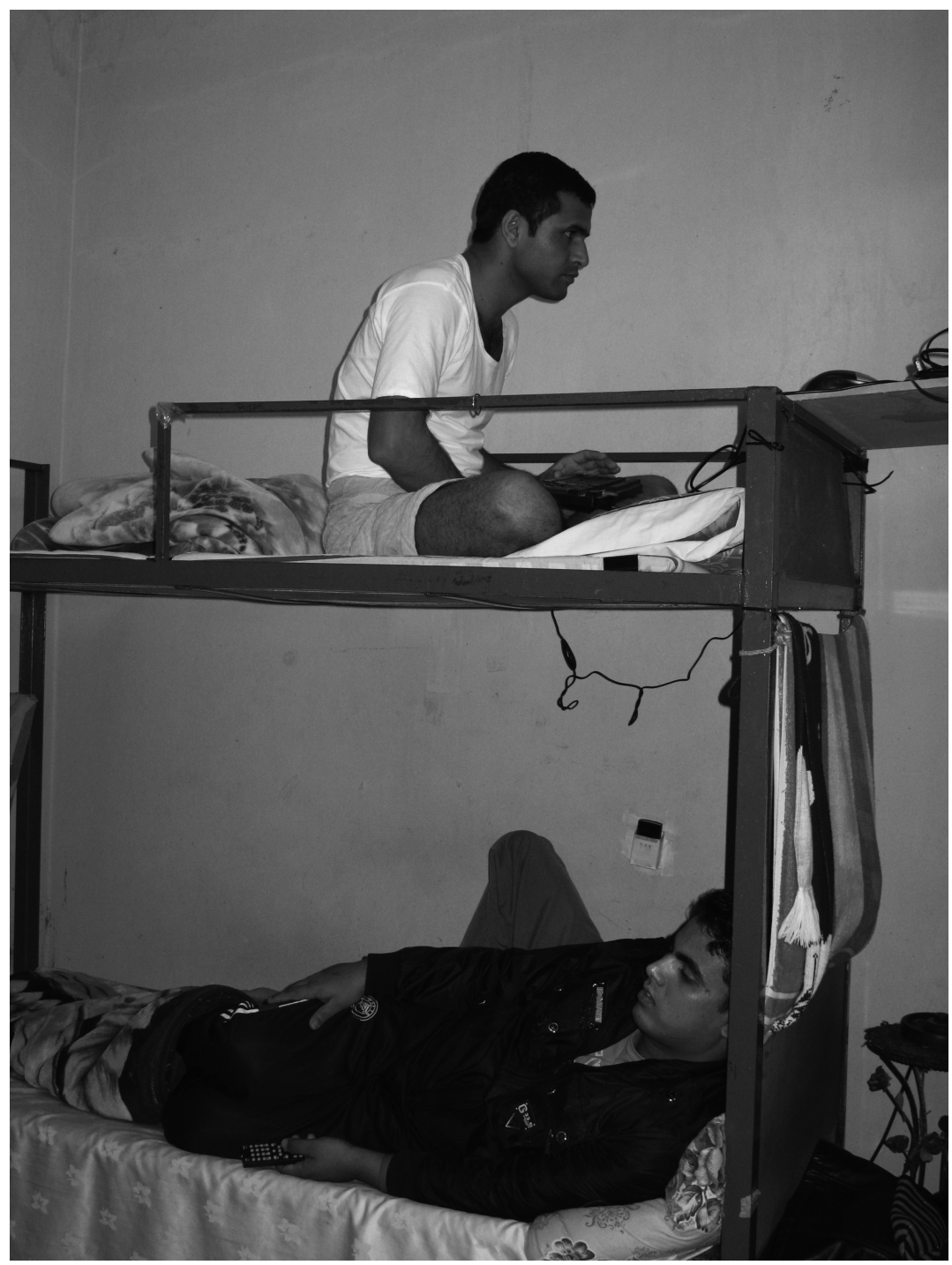

Photo 1 Le bricolage au service des nouvelles technologies (T. Bruslé) Do-it-yourself technologies assisting new technologies (T. Bruslé)

aux tons vifs, l'est plus. D'une manière générale, les murs collectifs, au sens où le regard n'est pas bloqué par un lit ou par la niche que forme le lit inférieur, sont peu décorés. Dans toutes les chambres, deux objets sont néanmoins présents. 
Sur la porte ou à proximité, un calendrier népalais ${ }^{22}$, généralement une réclame d'une banque ou d'un hôtel, est affiché, non loin d'une horloge à l'heure qatarie. Ces deux objets sont le signe que les séjours des migrants s'inscrivent à la fois dans le temps long de l'exil (celui du contrat, des vacances, du retour au pays) et dans le temps court de l'entreprise. Se tenir à un horaire fixe est bien souvent une chose apprise au Qatar, l'emploi et le contrôle du temps étant une forme de discipline des corps (Foucault, 1975).

Mais la décoration la plus affirmée, qui ne concerne cependant pas tous les hommes, relève plus du domaine privé. Autour de leur lit, les plus jeunes, non mariés en général, affichent des photos de vedettes népalaises ou indiennes. Les autres, des photos de leur famille. Il n'existe pas une volonté de décoration démonstrative d'un statut social comme dans le cas des mineurs en prison observés par Baer (2005). Ceux-ci exposent des désodorisants d'intérieur, des bouteilles de shampoing et autres produits d'hygiène pour affirmer leur identité personnelle et montrer des signes de richesse dans le cadre de l'économie informelle de la prison (ibid.). La décoration personnelle semble ici plus tourner vers l'espace mental intime des migrants, à quelques exceptions près. Contrairement à ce que décrit Baer, l'espace personnel dans les camps ne devient que rarement la maison et la décoration n'a pas fonction de remplir l'espace. Entre images de la modernité (buildings, voitures), drapeaux népalais, carte du pays ou photos de personnages de l'ex-royauté, la décoration permet de personnaliser l'espace pour le faire sien tout en gardant active la mémoire de la vie au pays, vie à la fois rêvée mais aussi source de sa nouvelle condition de migrant. Au regard des autres enfin, la décoration, car elle est aussi bricolage, est le moyen de dire quelque chose de soi (Lévi-Strauss, 1962) et d'affirmer son autonomie dans un monde où elle est rarement souhaitée.

\subsubsection{Prendre place dans l'espace, $c^{\prime}$ est s'affirmer}

La manière de s'approprier l'espace, par les meubles ou la décoration est aussi une certaine indication du pouvoir, sinon de l'aura du migrant. À l'arrivée dans le camp ou dans la chambre, il n'existe pas de rituel de dépossession. Les hommes entrent dans leur dortoir et prennent possession de leur lit avec le peu de bagages qu'ils ont. L'enjeu est donc plus de l'ordre de l'appropriation, même si la difficulté à établir des frontières personnelles en milieu clos (Sibley et Van Hoven, 2009) n'est pas établie de la même manière en prison que dans un camp de travailleurs. L'aménagement intérieur laisse libre cours à l'imagination, sans que des contrôles ou des restrictions viennent perturber les volontés inventives des hommes. Le dortoir est aussi l'espace où l'on peut s'exprimer librement, alors que toute autre forme d'expression est bannie ${ }^{23}$. Les tactiques des dominés (de Certeau, 1990)

22 Le calendrier lunaire népalais, vikram sambat, est en avance de cinquante-sept ans sur le calendrier grégorien.

23 Au Qatar, les droits politiques et syndicaux sont inexistants. Le droit du travail des travailleurs migrants ne permet pas de changer de travail à sa convenance, ni de former une organisation syndicale. Les 
sont de petites résistances, des arrangements qui ne contestent que peu l'ordre imposé par les employeurs et par l'État. La capacité d'action sur l'espace est limitée mais bien réelle. Elle se marque aussi par l'emploi du rideau de lit, qui est un dénominateur aux espaces de forte densité, où l'intimité est difficile à trouver. Dans l'espace transparent de la prison, l'espace personnel du lit protège (Sibley et Van Hoven, 2009). L'interdiction d'utiliser les draps comme rideaux dans les prisons russes est d'ailleurs un moyen d'accentuer la surveillance et de réduire l'intimité des prisonnières (Moran, Pallot, et Piacentini, 2013). Dans les dortoirs des prisons sud-africaines, le rideau, en bouchant la vue, signifie l'espace privé et limite la «maison » décorée à sa convenance (Dirsuweit, 1999). Dans le camp, il dit moins l'espace privé, tant le rythme du camp imprime une dichotomie privée/publique dans les usages de l'espace.

\subsection{Temps et usages du dortoir}

Si l'objet premier du dortoir est le sommeil, de nombreuses autres activités s'y déroulent en dépit de la forte densité et des contraintes spatiales et temporelles qui conditionnent la vie des hommes. Les temporalités d'usages du dortoir sont en effet totalement liées au rythme du camp, lui-même calqué sur les allers-retours des hommes impulsés par les besoins de l'entreprise ${ }^{24}$. De manière générale, les hommes quittent le camp le matin vers 5-6 heures et le retrouvent l'après-midi vers 15-16 heures. Certains se destinent alors à un deuxième emploi, comme domestiques dans un espace résidentiel fermé situé à proximité, où les cadres de leur entreprise sont logés. Le camp, et les dortoirs, se remplissent et se vident donc selon un rythme qui signifie en grande partie la dépossession du contrôle sur leur vie. Le vendredi est l'unique jour férié de la semaine, celui pendant lequel «l'entassement se fait le plus sentir».

\subsubsection{Le dortoir en autogestion}

Le dortoir fait très peu l'objet de contrôles de la part des encadrants. Une sorte d'autorégulation existe dans les chambres, où il n'y a pas de responsable attitré. Quand les hommes partent travailler, chacun range son lit, les couvertures sont pliées. Aucun objet ne traîne au centre de la pièce. Il faut respecter son tour de ménage - l'aspirateur est passé chaque jour -, ne plus parler ni allumer la lumière le soir après dix heures. Le partage de ces tâches, ainsi que la vie quotidienne, ne semble pas donner lieu à des tensions ${ }^{25}$. C'est sans doute lié au fort sens de la hiérarchie basée sur l'âge en cours chez les Népalais, qui se manifeste par le vouvoiement et une certaine soumission des plus jeunes vis-à-vis des anciens. Mais le camp n'est pas pour autant un lieu sans conflit, notamment car l'intermédiaire

mouvements spontanés de grève se soldent généralement par l'expulsion sine die des grévistes dans leur pays.

24 Voir Bruslé (2012).

25 Je ne saurai généraliser à tous les camps, vu que les journaux ont rapporté des cas de meurtres entre occupants de labour camp. 
principal entre l'entreprise et les employés privilégie les hommes de son propre réseau affinitaire. Ainsi il sélectionne "ses propres hommes» pour les postes les plus faciles et les mieux rémunérés et irait même jusqu'à se faire monnayer les demandes de congés. Les rapports de domination existent au sein même du groupe de travailleurs.

\subsubsection{Gérer la distance dans le dortoir}

De la même manière qu'il faut se déchausser en pénétrant dans une maison villageoise népalaise, on vit pieds nus dans le dortoir : la porte est un seuil qui marque la différence entre le couloir public et l'espace plus privé de la chambre. Dans la chambre, les hommes sont rarement seuls, surtout au retour du travail et le vendredi. Une fois retiré l'uniforme de travail, il s'agit de s'occuper.

La prise de possession de l'espace de la chambre se fait certes par les meubles et les objets mais aussi par une occupation de l'espace par les corps. Il faut se faire une place dans le dortoir, qui accueille souvent plus que les dix personnes qui y vivent. Les hommes se répartissent-ils dans la chambre pour maximiser la distance entre eux ? La gestion de la distance, telle qu'elle est pratiquée par les Népalais ne reposent pas uniquement sur l'éloignement. La question des limites entre les espaces et entre les hommes ne se pose pas comme l'ont remarqué Sibley et Van Hoven (2009) dans les dortoirs des prisons. Il n'est pas vraiment question de se protéger d'une contamination potentielle par les autres migrants. En effet, les corps se touchent, sans gêne et sans ambiguiité. À trois allongés sur un lit, ils regardent un film sur un ordinateur portable. Assis par terre, les épaules se touchent pour observer ensemble le dernier téléphone acheté. Pour regarder la télévision, certains s'allongent sur un lit, s'y assoient en tailleur tandis que d'autres prennent la même position par terre. Les corps sont à touche-touche mais quand le besoin d'intimité, toute relative néanmoins, se fait sentir, quand chacun souhaite être seul, la verticalité s'impose : l'un s'assoit à terre, l'autre sur le lit inférieur et le dernier sur le lit supérieur (Photo 2). D’une manière générale, les lits du bas sont ouverts, sauf lorsqu'il est explicitement écrit qu'il faut « demander avant de s'asseoir sur ce lit ».

Deux moments collectifs rassemblent presque tous les occupants d'une chambre. Les matches de football et de cricket réunissent les hommes autour de la télévision : chacun s'assoit comme il peut mais éviter la promiscuité n'est pas la préoccupation de tous (Photo 3). Le deuxième cas de communion collective, le plus banal, est le repas. Si les repas sont servis tous les soirs à la cantine (mess), les hommes sont peu nombreux à rester dans cette pièce froide meublée de tables et chaises en plastique. Ils rapportent en général leur assiette dans leur chambre ou y cuisinent dans un coin s'ils veulent sortir de l'ordinaire riz-lentille-curry de légumes ${ }^{26}$. Pendant que le riz cuit dans l'autocuiseur, l'un coupe les légumes sur une planchette tandis qu'un autre prépare le chutney dans un pétrin. Quand

26 En fait, il s'agit plutôt de cuisiner à son goût le même plat, et surtout de la viande. Les critiques envers la nourriture du camp sont constantes. 


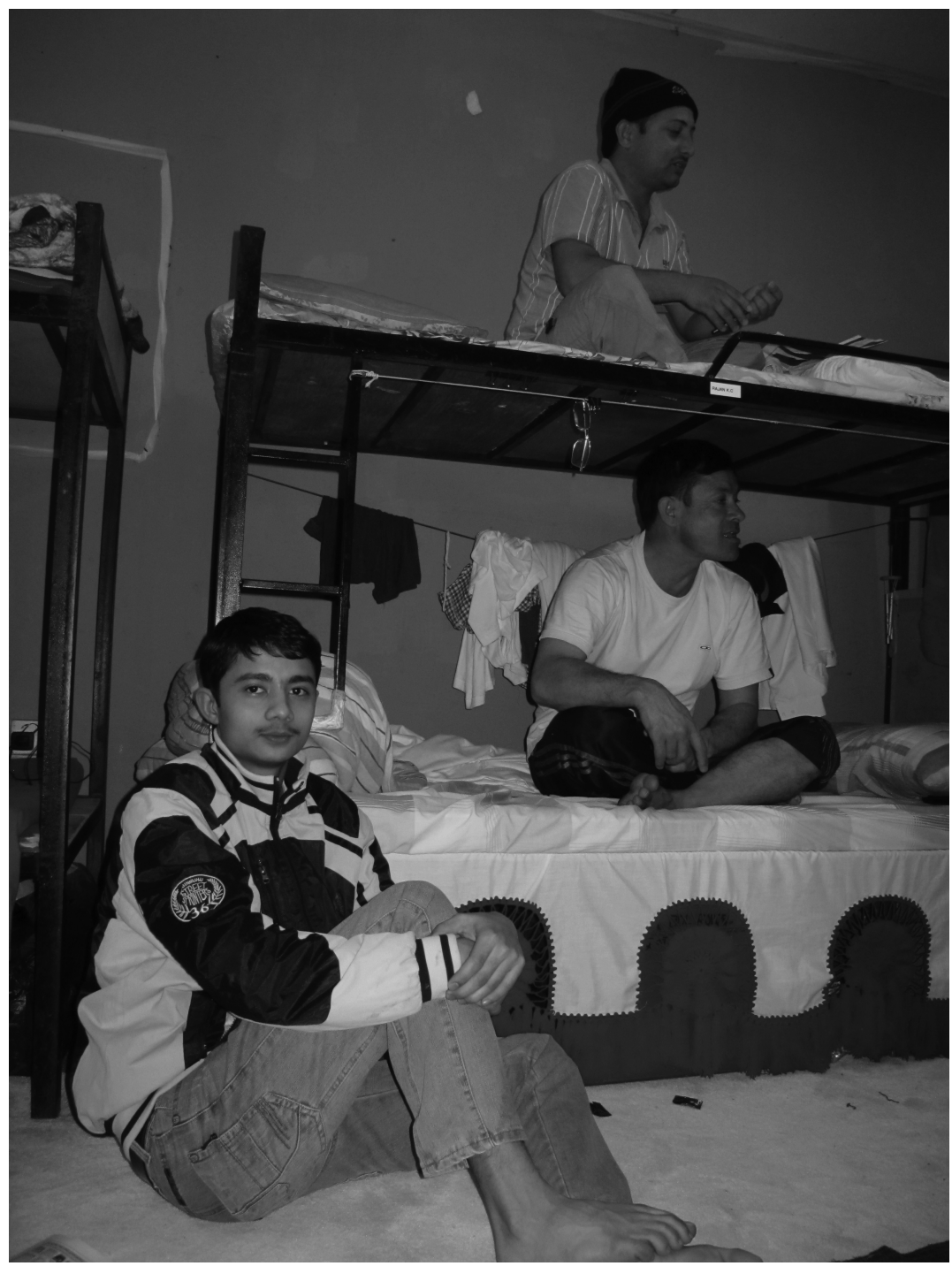

Photo 2 La verticalité pour gagner de l'espace (T. Bruslé)

Verticality used to improve space management (T. Bruslé)

tout est prêt, les hommes s'installent alors par terre, posant leur assiette sur une feuille de papier journal. Les jambes en tailleurs, le repas se consomme de la main droite, et très rapidement. On laisse une assiette pour celui qui rentre plus tard. Le dortoir impersonnel, parenthèse dans la vie des hommes, devient un espace de partage - les ustensiles et la nourriture ont été achetés en commun - et prend des traits domestiques. Il se fait salle à manger avec cependant une différence 


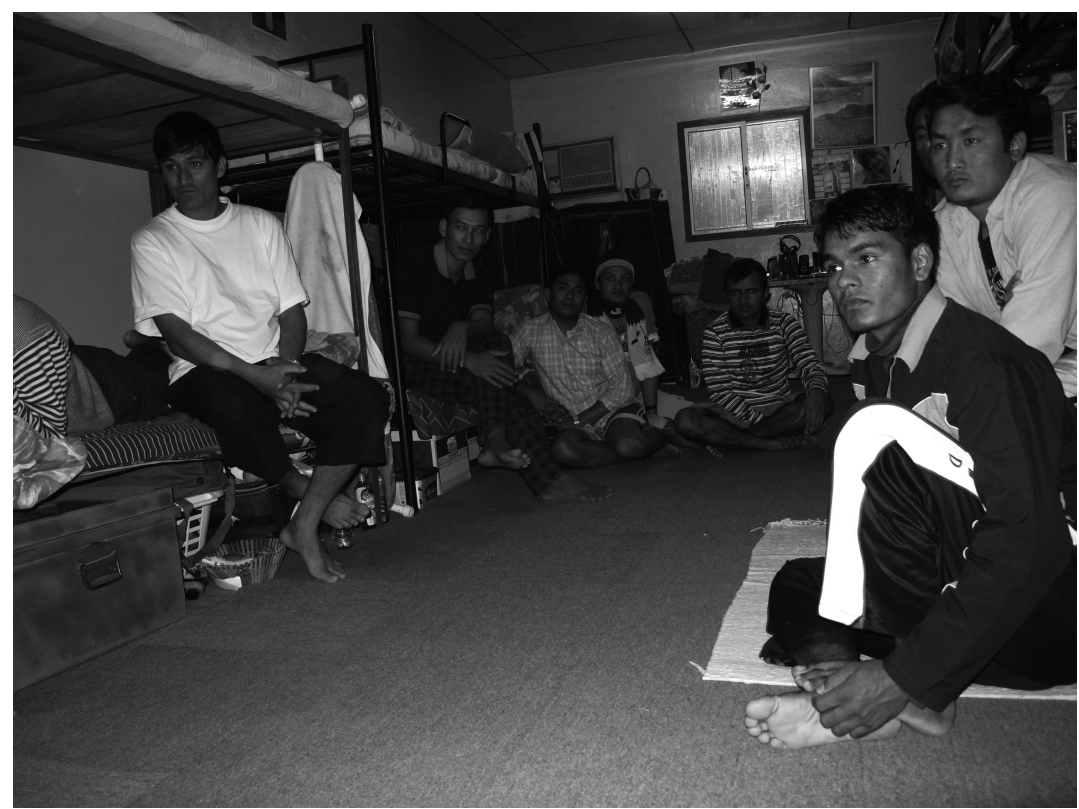

Photo 3 Un soir de coupe d'Asie de football (T. Bruslé) Watching the Asian football cup (T. Bruslé)

notoire par rapport au Népal rural : toutes les castes mangent ensemble ${ }^{27}$. Il n'en reste pas moins que l'ambiance fait penser à une atmosphère de commensalité népalaise. Pendant que les dernières productions cinématographiques népalaises tournent en boucle sur les ordinateurs ou à la télévision, les hommes dégustent le plat national parfois agrémenté de beurre clarifié rapporté du village par l'un d'eux. Dans une zone industrielle qatarienne, le dortoir d'un camp devient un univers domestique népalais, où la nourriture prend une place de choix.

L'espace du dortoir montre que la dichotomie espace privé/espace public est calée sur les activités biologiques et sociales et qu'elle joue aussi sur des micro-espaces tel le lit $^{28}$. Le même lieu change de statut en fonction du rythme du camp et des activités effectuées.

\subsubsection{Passer le temps : un habiter de l'ennui}

Entre seize heures et l'extinction des feux, les chambres sont pleines, comme le vendredi. C'est le moment où chacun retrouve une liberté d'usage de son temps mais aussi le moment où il faut le «tuer »: « ici le temps ne passe pas, une heure

27 Les membres des basses castes, qui pourraient souffrir de discriminations basées sur la caste, reconnaissent l'absence de comportements dérogatoires envers eux. L'observation des repas et de l'eau partagés confirme leurs dires.

28 Cela a aussi été noté par Moran, Pallot et Piacentini (2013) dans les prisons pour femmes en Russie. 
dure comme deux heures ». Tout comme en prison où passer le temps est l'un des problèmes majeurs (Milhaud 2009), le temps libéré, celui qui n'est pas dévolu à des fonctions de production, doit être occupé. Il faut lutter contre l'ennui d'une vie vécue comme une obligation, dans un environnement où les déplacements sont fortement contraints. Les divertissements dans le camp sont peu nombreux. La zone industrielle n'en offre aucun, sinon une petite zone commerciale, un supermarché et un restaurant népalais. Dans le camp même, quand il ne fait pas trop chaud, ou le soir à la lumière de projecteurs, des parties de volley-ball sont organisées. Ceux qui ne font pas partie de l'association culturelle se retrouvent dans les dortoirs où ne pas faire grand-chose, discuter, repasser son linge, ranger ses affaires sont les activités qui permettent de tromper l'ennui. Les nouvelles technologies ont un attrait indéniable sur les hommes et permettent non pas tant une ouverture sur le monde que de rester connecté à la culture populaire népalaise et de créer un lien avec l'extérieur (Bruslé, 2012b). C'est aussi dans ces moments-là que les circulations entre chambres sont les plus élevées. A priori les dortoirs sont ouverts à tous, mais on constate que les plus récemment arrivés, les plus jeunes, ne sortent que peu de l'espace qui leur a été affecté. Au contraire, les plus charismatiques du camp, ceux qui parlent fort et n'hésitent pas à s'imposer, entrent à leur guise où ils veulent. On leur laisse la chaise pour s'asseoir, comme au chercheur de passage. Mais on ne peut en tout cas définir le dortoir comme un espace privé car il n'est entre autre pas caractérisé par sa clôture (Staszak, 2001). Malgré une certaine fermeture, plus de l'ordre de l'intériorité que de règles communes, les portes des chambres sont ouvertes et le restent même durant la journée. La solidarité et la confiance entre les hommes sont telles que les portefeuilles ou les ordinateurs portables sont rangés sous les oreillers, sans crainte de vol.

Enfin, la proximité et l'impression de densité dans le camp ne se limitent pas au touché. En fin de journée et le vendredi, la télévision, les sonneries de téléphone, les conversations, les vidéos YouTube ou la ventilation de l'air conditionné emplissent les dortoirs. La nuit n'est pas non plus gage de silence, tant les divers bruits de moteurs (pompes, climatisation) et les grincements des lits métalliques forment un paysage sonore saturé.

\section{Le dortoir, un espace dévalorisé}

Si vivre dans un camp est une expérience exceptionnelle à l'arrivée au Qatar, elle devient assez rapidement banale. Le dortoir représente alors le lieu d'une communauté de destin que les migrants partagent $\mathrm{t}^{29}$. À cause de la contraction des possibles en terre qatarienne, la majorité des hommes se font une raison de leur vie de migrant à la mobilité réduite et s'installent dans une routine qui ne les

29 L'immense majorité des hommes qu'ils connaissent au Qatar vivent aussi dans des camps de travailleurs. 
fait que peu sortir du camp hors du travail ${ }^{30}$. Cette situation et l'impression de séjour forcé, telles qu'elles sont ressenties par les migrants, instillent des valeurs liées à la prison au moment migratoire (Bruslé, 2012a). Qu'en est-il alors du dortoir comme espace de base de la vie quotidienne ? Quelles sont les valeurs que les hommes lui attribuent ? On peut s'interroger sur les rapports entre le dortoir comme base spatiale de la vie quotidienne et l'identité de migrant, en se demandant si les espaces domestiques, dont les dortoirs du camp partagent certains traits, sont porteurs de normes et de valeurs et sources d'identification (Staszak, 2001). La discussion sur le dortoir comme maison doit dépasser les pratiques, qui remplissent les fonctions de l'habitat domestique. Il faut s'intéresser à ce qu'en disent les hommes.

\subsection{Le dortoir fait-il le migrant ?}

Moran et al. (2013) décrivent l'intimité comme un ensemble de relations émotionnelles mais aussi comme un lieu où l'on retrouve son "soi » grâce à la solitude ${ }^{31}$. En migration, la question de l'individualité peut aussi être posée, tant l'expérience migratoire change l'individu. L'absence d'espace privé, particulièrement dans les conditions extrêmes de sur-occupation des chambres et des lits dans les logements pour migrants en Afrique du Sud, signifie le déni de la personne (Ramphele, 1993). Alors que les structures nient l'individualité des migrants pour en faire des groupes nationaux homogènes, selon le principe de "l'illusion communautaire »(Sayad, 1980, 95), les hommes tentent néanmoins de se construire comme sujets, par la décoration ou les objets possédés. Le fait que le logement soit à la fois contraint et vu comme temporaire ("Nous sommes là pour deux jours ») confirme néanmoins que l'espace domestique « est un miroir dans lequel on peut voir les structures et les valeurs essentielles d'une société donnée » (Staszak, 2001, 348). Les décorations dans le dortoir reflètent une micro-géographie de la mémoire et de l'émotion transnationales. Le lien au pays, à la vie devenue anormale puisque la migration est la norme, se matérialise par des photos de familles ou de paysages. Le migrant est présent ici, absent de là-bas et les photos lui rappellent sans cesse sa condition d'exilé. Mais dans la décoration, la manière de l'habiter, de s'y montrer, il y a aussi un ensemble de normes et de valeurs qui se diffuse, et qui font partie d'une «culture de la migration »(Cohen et Sirkeci, 2011). Le dortoir est le lieu de base d'une vie de souffrance ${ }^{32}$ : c'est une vie difficile, une vie d'éloignement, où l'on ne fait pas fortune, mais "c'est notre vie ». La vie dans le camp n'est pas difficile en elle-même car la solidarité, l'impression de partager un destin avec les autres, la rend banale. Le dortoir n'est certes pas l'espace fondamental, il n'est pas le plus chargé ni ne forme la base spatiale d'une quelconque appartenance. N'empêche qu'il est un espace de vie, et de vie prolongé. Il est porteur d'identité sociale,

30 L'expérience migratoire est souvent résumée par cette phrase : « on ne vient que pour le travail ».

31 Goffman (1968) insiste aussi sur les changements de personnalité induite par l'enfermement.

32 En népali, le terme dukh (souffrance, peine) revient constamment pour décrire la condition de migrant. 
même minimale, celle de migrant. Il est chargé émotionnellement : c'est l'espace de la séparation d'avec la famille, de l'éloignement, du travail, de la souffrance, de l'abnégation mais aussi de l'émancipation et d'une certaine modernité. C'est aussi celui grâce auquel son rôle d'homme responsable d'une famille va pouvoir se réaliser. Ainsi, c'est un espace de projection dans le futur, fondamentalement temporaire (bien que le temps de l'expatriation dure plus ou moins longtemps). Le soi exhibé est celui d'un homme qui ne peut vraiment s'affirmer par les lieux, le dortoir et le camp étant dévalorisants ${ }^{33}$. Les quelques-uns qui ont obtenu des diplômes au Qatar en remerciement « du soin constant et inconditionnel apporté par Monsieur X à son travail » les affichent tout de même comme une reconnaissance. Mais pour autant, être migrant, c'est « devenir le serviteur ou l'esclave de son employeur », donc foncièrement dégradant.

\subsection{Le dortoir, un lieu par défaut dans une vie de contraintes}

Placé sous le signe de l'obligation, le séjour au Qatar est vécu comme une suite de contraintes ${ }^{34}$. Dès lors, les lieux imposés sont porteurs de valeurs négatives. Les zones industrielles «ne sont pas faites pour les êtres humains » et la vie au Qatar est une vie de prisonniers. Pourtant, le dortoir, " où il n'y a pas d'espace personnel », est le seul endroit où se tenir. Même s'il n'est pas l'espace de la maison, encore moins celui de la famille, il est un espace du repli face à l'hostilité de la société qatarienne, à la difficulté d'accès aux lieux communs de Doha ou au manque d'intérêt du monde du travail. Bien qu'il soit « inconfortable », le dortoir apporte néanmoins une certaine protection. De toute façon, la résignation par rapport à sa condition de travailleur migrant s'applique aussi aux conditions de vie : "quand on habite dans une chambre (room), on n'est pas tout seul, il n'y a pas d'endroits personnels. Au début, pendant un ou deux jours, c'est difficile. Ensuite, là où on habite, il faut s'y faire, c'est bien ». De fait, les hommes finissent par apprécier leur propre dortoir. L'appartenance temporaire au dortoir, qui s'entend dans quelques expressions ("notre chambre »), qui sert aussi à situer les hommes ( Rajan de la chambre $n^{\circ} 6$ ») n'est pourtant pas synonyme d'identification. Le lieu dortoir ne se fait pas territoire. Le dortoir, et plus généralement le camp et le Qatar sont considérés comme des étapes dans la vie des migrants, donc comme un hiatus.

On peut néanmoins noter que certains, peu nombreux, souvent ceux qui sont depuis une longue période au Qatar, dans le même dortoir et ont sans doute réussi leur projet migratoire, attribuent au camp et au dortoir des valeurs domestiques. "Ici, nous ne sommes que des frères népalais, on habite ensemble et on s'entend bien. C'est pourquoi on a l'impression d'être à la maison. Si on

33 Sur Facebook par exemple, il est extrêmement rare que les hommes postent des photos de la vie dans le camp.

34 La notion d'obligation à partir travailler, pour nourrir les siens, pour envoyer ses enfants à l'école ou pour acheter de la terre - en bref pour construire un futur à sa famille -, est omniprésente quand les migrants évoquent des raisons de leur venue au Qatar. 
est dix dans une chambre, on vit dans la même chambre, comme dans la même maison. Tous, on mange la nourriture de celui qui l'a préparée, on dort ensemble, on s'amuse ensemble. Le vendredi, c'est romantic » dit Ram qui a passé plus de huit années dans le camp. Ce à quoi Ramesh ajoute : "à force de rester ici, le camp devient comme une maison, un village. Pour les nouveaux, c'est plus dur, ils ne savent pas que faire, comment faire ».

Enfin, contrairement à ce qui se passe en Chine du Sud où le système du logement en dortoirs facilite la mobilisation politique des hommes sur une base de classe, et non plus sur des bases ethniques ou régionales (Chan et Pun, 2009), le dortoir au Qatar n'est pas un espace de revendication politique. Même s'il a pu exister des révoltes d'ouvriers au Qatar - en 2006 une rébellion importante a eu lieu à Ras Laffan en réaction à la mort d'ouvriers dans leur sommeil, tous les hommes ont été expulsés - ou à Dubaï (Buckley, 2013), même si le camp est là où se fomentent les protestations, le camp ne me semble pas être un lieu de la rébellion. Les rapports de domination reposent sur des relations interpersonnelles entre l'entreprise ou son représentant dans le camp et les migrants. En outre, la résistance est plus fragile, moins politique, et plus centrée sur le bien-être et l'espace intérieur.

\section{Des logements sans qualité pour des hommes sans qualité}

Le titre de cette conclusion reflète le double point de vue de l'État qatarien sur les travailleurs et des migrants eux-mêmes vis-à-vis de leur logement.

Les spatialités du pouvoir de l'État constituent un «assemblage de pratiques spatiales » (Martin et Mitchelson, 2009, 470) fruit d'une idéologie du contrôle social, alimenté par la méfiance envers certains groupes (Foucault, 1975 ; Wacquant, 2007). Au Qatar, la catégorisation des célibataires sud-asiatiques comme groupe non désirable a des conséquences directes sur leur vie quotidienne, en particulier par la création d'enclaves résidentielles éloignées des centres-villes. Les productions discursives qui stigmatisent une catégoriale ethno-sociale trouvent leur expression spatiale dans la relégation, l'invisibilisation et la déshumanisation architecturale. Comme le foyer pour travailleurs en France, le camp «n'est pas un sas d'entrée vers le territoire national, mais une enclave temporaire fondamentalement tournée vers l'extérieur » (Bernardot, 2008), l'extérieur étant ici les pays d'origine des migrants. Le provisoire est donc ancré dans la conception de l'ouvrier migrant et de son logement. Si l'objectif du camp et du dortoir est la fabrication de « corps dociles » (Foucault, 1975), utiles à l'économie et obéissants politiquement, il est à peu près atteint. Les forces de contestation sont cantonnées au camp, les résistances se font à l'échelle micro-géographique du bricolage. Le migrant en tant qu'éventuel citoyen ou homme doté d'un pouvoir politique est quasiment réduit à néant. Il est uniquement devenu un corps domestique dans le camp et un corps productif au travail. 
Le camp comme institution d'enfermement, loin d'un contrôle total sur les hommes, permet néanmoins des ajustements. Le logement est un instrument de contrôle et de relégation mais il peut faire l'objet d'une appropriation. L'étude micro-géographique des espaces intérieurs montre que la production d'espaces dans les coins et les recoins participent sinon de résistances du moins d'une " géographie de la liberté » (Goffman, 1968, 286). La géographie de l'intérieur est ainsi capable de s'intéresser aux lieux non identificatoires, rejoignant en cela les non-lieux d'Augé (1992) mais en dépassant le caractère transitoire, banal et ubiquiste des espaces décrits par cet auteur. En situation d'enfermement, l'appropriation de l'espace est provisoire : l'habiter contraint est un habiter bricolé et temporaire. Dans le dortoir, l'espace est plus produit par les interactions horizontales entre les hommes que de manière verticale par le contrôle de l'institution comme c'est le cas en prison (Sibley et Van Hoven, 2009). La nature profondément spatiale des "manières de faire » (de Certeau, 1990) dans une chambre où logent huit à dix personnes nous montre que les processus d'appropriation, de privatisation, de bricolage sont nécessaires à une existence prolongée dans des lieux non choisis, éloignés des réseaux familiers. Le dortoir, lieu de vie principal en dehors du travail, est l'échelle des interactions tandis que les temporalités des usages du lit montrent une utilisation fine de l'espace. Pour échapper à une situation contrainte (obligation de migrer, d'habiter dans un logement collectif, perte de maitrise du temps), les hommes (mais pas tous) mobilisent des ressources relationnelles (les amis), financières (achat d'ordinateurs) et spatiales (les recoins de la chambre ou le lit). La frugalité des intérieurs se transforme progressivement en une abondance contenue, qui donne lieu à des négociations et arrangements spatiaux, donc à une véritable économie de la rareté spatiale dans le dortoir. "La tactique n'a pour lieu que celui de l'autre » (de Certeau, 1990, 87) : dans les lieux de l'autre que sont le camp et le dortoir, prendre place dans l'espace est une affaire de temps, de prestige mais aussi d'affirmation personnelle. Les espaces d'autonomie et d'affirmation sont à conquérir, non pas selon un processus conflictuel, mais selon des arrangements entre les hommes, tant les espaces du dortoir et les objets sont partagés. Le conflit est évité par la soumission aux plus anciens et par l'intérêt relatif à posséder son propre espace. Bien que souvent ténue car tous ne participent pas à la personnalisation du dortoir, "l'empreinte visuelle des tactiques » (Baer, 2005, 214) est aussi spatiale, à l'échelle du lit ou recoin. Les tactiques s'expriment spatialement, dans la durée pour les décorations, dans l'instant pour ce qui est de la prise de l'espace par les corps. Le dortoir permet de se retrouver, de retrouver une liberté et une affirmation de soi. Mais ce que les hommes bricolent avec l'espace et les objets ne sont que de faibles résistances en regard du contrôle de leurs mouvements qu'ils subissent.

Par sa temporalité spécifique et son mode contraint, habiter un camp de travailleurs est en marge par rapport à l'habiter classique. Même s'il peut sembler inhabitable au départ, le camp de travailleur est néanmoins approprié et porteur de valeurs. Face aux peu de possibilités d'adaptation, les manières d'habiter le camp 
sont homogènes parmi les hommes. Le dortoir n'est pas un espace domestique. S'il participe à la fabrication du migrant, il ne représente bien souvent qu'un espace de l'obligation qui n'est habité que pour être quitté. Le dortoir est le lieu du présent, mais d'un présent transitoire, qui devrait permettre un avenir meilleur. En ce sens, l'investissement minimal dans le lieu et l'esthétique de la récupération qui dominent les paysages intérieurs confortent finalement les politiques qatariennes de gestion de la main-d'œuvre. Les migrants sont présents de manière provisoire, ils partiront une fois les grands travaux terminés et/ou une fois leur projet migratoire atteint.

Centre d'études himalayennes

7 , rue Guy Môquet

94801 Villejuif CEDEX

Tristan.brusle@vjf.cnrs.fr

\section{Bibliographie}

Adham K. (2008), "Rediscovering the Island: Doha's Urbanity from Pearls to Spectacle », in Y. Elsheshtawy (dir.), The Evolving Arab City. Tradition, Modernity and Urban Development, Londres et New York, Routledge, p. 218-257.

Augé M. (1992), Non-lieux. Introduction à une anthropologie de la surmodernité, Paris, Seuil, 150 p.

Baer L. D. (2005), « Visual Imprints on the Prison Landscape : A Study on the Decorations in Prison Cells », Tijdschrift Voor Economische En Sociale Geografie, 96, 2, p. 209-217.

Baldwin-Edwards M. (2011), Labour Immigration and Labour Markets in the GCC Countries : National Patterns and Trends. Kuwait Programme on Development, Governance and Globalisation in the Gulf States, Londres, LSE, 71 p.

Beaugrand C. (2010), " Politiques de non-intégration dans les monarchies du golfe », Transcontinentales, 8/9, http:// transcontinentales. revues. org/793.

Bernardot M. (2007), "Loger et déplacer les indésirables. Sociologie du logement contraint », in C. Levy-Vroelant (dir.), Logement précaire en europe. Aux marges du palais, Paris, L'Harmattan, p. 286-303.

Bernardot M. (2008), "Camps d'étrangers, foyers de travailleurs, centres d'expulsion : les lieux communs de l'immigré décolonisé », Cultures \& Conflits, n 69, p. 55-79.

Bruslé T. (2010), «Who's in a Labour Camp ? À Socio-economic Analysis of Nepalese Migrants in Qatar », European Bulletin of Himalayan Research, n³5-36, p. $154-170$.

Bruslé T. (2012a), " Daily Life, Privacy and the Inmate Metaphor in a Nepalese Workers'Labour Camp (Qatar) ", South Asian Multidisciplinary Academic Journal, nº 6, http://samaj.revues.org/3446.

Bruslé T. (2012b), "What's New in the Gulf? New Technologies, Consumption and Display of Modernity Among Nepalese Workers in Qatar », E-migrinter, n 8, p. 59-73.

Buckley M. (2013), « Locating Neoliberalism in Dubai : Migrant Workers and Class Struggle in the Autocratic City», Antipode, 45, 2, p. $256-274$.

Caraher W., Weber B. et Kourelis K. (à paraître), « North Dakota Man Camp Project : The Archaeology of Home in the Bakken Oil Fields », manuscript non publié, Grand Forks, University of North Dakota.

Castles S. (2006), "Guestworkers in Europe : A Resurrection? » International Migration Review, 40, 4, p. $741-766$. 
Chan C.K.C. et Pun N. (2009), "The Making of a New Working Class ? À Study of Collective Actions of Migrant Workers in South China », China Quarterly, n 198, p. 287 - 303.

Cohen J.H. et Sirkeci I. (2011). Cultures of Migration. The Global Nature of Contemporary Mobility, Austin, University of Texas Press, 165 p.

Collignon B. et Staszak J.-F. (2003), Espaces domestiques. Construire, habiter, représenter, Paris, Bréal, $447 \mathrm{p}$.

Darley M. (2008), « Frontières, asile et détention des étrangers : le contrôle étatique de l'immigration et son contournement en Autriche et en République Tchèque ", Thèse de doctorat en sciences politiques, Paris, IEP Paris, $637 \mathrm{p}$.

De Certeau M. (1990), L'invention du quotidien. 1. Arts de faire, Paris, Gallimard, Collection Folio, $350 \mathrm{p}$.

Di Méo G. (1998), Géographie Sociale et Territoires, Paris, Nathan Université, 320 p.

Dirsuweit T. (1999), "Carceral Spaces in South Africa : a Case Study of Institutional Power, Sexuality and Transgression in a Women's Prison », Geoforum, 30,1, p. 71-83.

Foucault M. (1975), Surveiller et punir : naissance de la prison. Paris, Gallimard, 318 p.

Gardner A. (2010a), « Engulfed : Indian Guest Workers, Bahraini Citizens, and the Structural Violence of the Kafala System », in N. De Genova et N. Peutz (dir.), The Deportation Regime. Sovereignty, Space, and the Freedom of Movement, Durham \& London, Duke University Press, p. 196 - 223.

Gardner A. (2010b), "Labor Camps in the Gulf States », In Middle East Institute Viewpoints, Migration and the Gulf, Washington DC, The Middle East Institute, p. 55-57.

Goffman E. (1968), Asiles, études sur la condition sociale des malades mentaux et autre reclus, Paris, Éditions de Minuit, $451 \mathrm{p}$.

Heeg J. (2010), Seeing Security: Societal Securization in Qatar, Thèse de doctorat, Washington, Georgetown University, 320 p.

Human Rights Watch, (2012), Building a Better World Cup. Protection Migrant Workers in Qatar Ahead of FIFA 2022, Human Rights Watch, 152 p.

Lévi-Strauss C. (1962), La pensée sauvage, Paris, Plon, 395 p.

Martin L. et M. Mitchelson (2009), "Geographies of Detention and Imprisonment : Interrogating Spatial Practices of Confinement, Discipline, Law, and State Power », Geography Compass, 3, 1, p. 459-477.

Michalon B. (2012), «La mobilité au service de l'enfermement ? Les centres de rétention pour étrangers en Roumanie », Géographie et Cultures, 81, 9, p. 91-110.

Milhaud O. (2009). Séparer et punir. Les prisons françaises : mise à distance et punition par l'espace, Thèse de Doctorat de géographie, Bordeaux, Université de Bordeaux, 368 p.

Moran D., Pallot J. et Piacentini L. (2013), «Privacy in Penal Space : Women's Imprisonment in Russia », Geoforum, doi : 10.1016/j. geoforum.2013.01.002.

Morelle M. (2006), "La rue dans la ville africaine (Yaoundé, Cameroun et Antananarivo, Madagascar) 》, Annales de Géographie, n²650, p. 339-360.

Morelle M. (2013), "La prison centrale de Yaoundé : I'espace au cœur d'un dispositif de pouvoir », Annales de Géographie, n 691, p. 332-356.

Portes A. (1997), Globalization from Below: The Rise of Transnational Communities, Princeton University, $26 \mathrm{p}$.

Pun N. et Chan J. (2013), "The Spatial Politics of Labor in China : Life, Labor, and a New Generation of Migrant Workers », South Atlantic Quarterly, 112, 1, p. 179-190.

Pun N. et Smith C. (2007), «Putting Transnational Labour Process in Its Place : the Dormitory Labour Regime in Post-socialist China », Work Employment Society, 21, 1, p. 27-45. 
Ramphele M. (1993), A Bed Called Home. Life in the Migrant Labour Hostels of Cape Town, Athens, Ohio Press University, Athens, 152p.

Ripoll F. et Veschambre V. (2005), «L'appropriation de l'espace comme problématique », Norois, $\mathrm{n}^{\circ} 2$, p. 7-15.

Sayad A. (1980), "Le Foyer Des Sans-familles », Actes de La Recherche en Sciences Sociales, 32, 1, p. 89-103.

Sibley D. et Van Hoven B. (2009), "The contamination of personal space : boundary construction in a prison environment », Area, 41, 2, p. 198-206.

Staszak J.-F. (2001), "L'espace domestique : pour une géographie de l'intérieur », Annales de Géographie, n 620, p. 339-363.

Stock M. (2004), "L'habiter comme pratique des lieux géographiques », EspacesTemps. net, http://www.espacestemps.net/en/articles/Irsquohabiter-comme-pratique-des-lieuxgeographiques-en/.

Veschambre V. (2005), "La notion d'appropriation », Norois, n² 2, p. 115-116.

Vitalis R. (2009), America's Kingdom. Mythmaking on the Saudi Oil Frontier, London, New York, Verso, $353 \mathrm{p}$.

Wacquant L. (2007), "Territorial Stigmatization in the Age of Advanced Marginality », Thesis Eleven, $\mathrm{n}^{\circ} 91$, p. 66-77.

Wacquant L. (2010), "Designing Urban Seclusion in the Twenty-first Century », Perspecta, The Yale Architectural Journal, $\mathrm{n}^{\circ} 43$, p. 164-176.

Wiedmann F., Salama A.M. et Thierstein A. (2012), "Urban Evolution of the City of Doha : An Investigation into the Impact of Economic Transformations on Urban Structures », Middle East Technical University Journal of the Faculty of Architecture, 29, 2, p. 35-61.

Zeneidi D. (2011), « Migrations circulaires et déni de reconnaissance. L'expérience de I'injustice spatiale des saisonnières agricoles marocaines à Huelva (Espagne) », Justice Spatiale | Spatial Justice, $\mathrm{n}^{\circ} 3$, http://www.jssj.org. 